\title{
Transcriptional landscape in rat intestines under hypobaric hypoxia
}

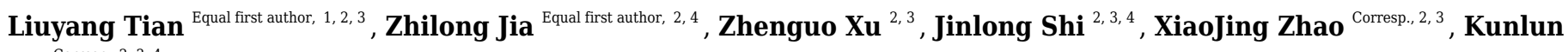 \\ He Corresp. 2, 3, 4 \\ ${ }^{1}$ School of Medicine, Nankai University, Tianjin, China \\ 2 Beijing Key Laboratory of Chronic Heart Failure Precision Medicine, Medical Innovation Research Division Of Chinese PLA General Hospital, Beijing, China \\ 3 Military translational medicine lab, Medical Innovation Research Division Of Chinese PLA General Hospital, Beijing, China \\ 4 Key Laboratory of Biomedical Engineering and Translational Medicine, Ministry of Industry and Information Technology, Medical Innovation Research \\ Division of Chinese PLA General Hospital, Beijing, China \\ Corresponding Authors: Xiaojing Zhao, Kunlun He \\ Email address: xjingzhao@126.com, kunlunhe@plagh.org
}

Oxygen metabolism is closely related to the intestinal homeostasis environment, and the occurrence of many intestinal diseases is as a result of the destruction of oxygen gradients. The hypobaric hypoxic environment of the plateau can cause dysfunction of the intestine for humans, such as inflammation. The compensatory response of the small intestine cells to the harsh environment definitely changes their gene expression. How the small intestine cells response the hypobaric hypoxic environment is still unclear. We studied the rat small intestine under hypobaric hypoxic conditions to explore the transcriptional changes in rats under acute/chronic hypobaric hypoxic conditions. We randomly divided rats into three groups: normal control group (S), acute hypobaric hypoxia group, exposing to hypobaric hypoxic condition for 2 weeks (W2S) and chronic hypobaric hypoxia group, exposing to hypobaric hypoxic condition for 4 weeks (W4S). The RNA sequencing was performed on the small intestine tissues of the three groups of rats. The results of principal component analysis showed that the W4S and W2S groups were quite different from the control group. We identified a total of 636 differentially expressed genes, such as ATP binding cassette, Ace2 and Fabp. KEGG pathway analysis identified several metabolic and digestive pathways, such as PPAR signaling pathway, glycerolipid metabolism, fat metabolism, mineral absorption and vitamin metabolism. Cogena analysis found that up-regulation of digestive and metabolic functions began from the second week of high altitude exposure. Our study highlights the critical role of metabolic and digestive pathways of the intestine in response to the hypobaric hypoxic environment, provides new aspects for the molecular effects of hypobaric hypoxic environment on intestine, and raises further questions about between the lipid metabolism disorders and inflammation. 


\section{Transcriptional landscape in rat intestines under hypobaric}

\section{2 hypoxia}

3 Liuyang $\operatorname{Tian}^{1,2,3, \#}$, Zhilong Jia ${ }^{2,4, \#}$, Zhenguo $\mathrm{Xu}^{2,3}$, Jinlong Shi ${ }^{2,3,4}$, Xiaojing Zhao ${ }^{2,3,{ }^{*}}$ and Kunlun He ${ }^{2,3,4, *}$

$4 \quad{ }^{1}$ School of Medicine, Nankai University, Tianjin, China

$5 \quad{ }^{2}$ Beijing Key Laboratory of Chronic Heart Failure Precision Medicine, Medical Innovation Research

6 Division of Chinese PLA General Hospital, China

$7 \quad{ }^{3}$ Military Translational Medicine Laboratory, Medical Innovation Research Division of Chinese PLA

8 General Hospital, Beijing, China

$9 \quad{ }^{4}$ Key Laboratory of Biomedical Engineering and Translational Medicine, Ministry of Industry and

10 Information Technology, Medical Innovation Research Division of Chinese PLA General Hospital, Beijing,

11 China

12 \#These authors contributed equally

$13{ }^{*}$ Corresponding Authors:

14 Xiaojing Zhao, Kunlun He

15 No.28 Fuxing Road, Beijing, China

16 Email address: xjingzhao@126.com and kunlunhe@plagh.org 


\section{Abstract}

Oxygen metabolism is closely related to intestinal homeostasis and many intestinal diseases are caused by the destruction of oxygen gradients. The hypobaric hypoxic environment of the medical plateau can cause dysfunctions, such as inflammation in human intestines and prompts small intestine cells to change their gene expression. How the small intestine cells change when exposed to the hypobaric hypoxic environment is still unclear. Rat small intestines were studied under hypobaric hypoxic conditions to explore the transcriptional changes in rats under acute/chronic hypobaric hypoxic conditions. The rats were randomly divided into three groups: normal control group (S), acute hypobaric hypoxia group (W2S), and chronic hypobaric hypoxia group (W4S). The W2S was exposed to hypobaric hypoxic conditions for 2 weeks and the W4S was exposed to hypobaric hypoxic conditions for 4 weeks. RNA sequencing was performed on the small intestine tissues of the three groups of rats. The principal component analysis results showed that the W4S and the W2S groups were quite different from the control group. A total of 636 differentially expressed genes were identified, such as ATP binding cassette, angiotensin I converting enzyme 2 (Ace2), and fatty acid binding protein (Fabp). Kyoto Encyclopedia of Genes and Genomes (KEGG) pathway analysis identified several metabolic and digestive pathways that were affected, such as the peroxisome proliferator-activated receptor (PPAR) signaling pathway, glycerolipid metabolism, fat metabolism, mineral absorption, and vitamin metabolism. Cogena analysis found that up-regulation of digestive and metabolic functions began from the second week of high-altitude exposure. This study highlights the critical role of the metabolic and digestive pathways of the intestine in response to the hypobaric hypoxic environment, provides new information about the molecular effects of hypobaric hypoxic environment on the intestine, and raises further questions about the relationship between lipid metabolism disorders and inflammation.

\section{Introduction}

Quick entry into the hypoxic environment of the medical plateau, defined as an area located 3000 meters above sea level, can lead to serious damage to the organs of the body (Taino et al. 2019). In high-altitude areas, oxygen content and oxygen partial pressure decrease, forming a hypobaric hypoxic environment. Hypobaric hypoxia can lead to insufficient arterial blood oxygen content; this phenomenon is called systemic hypoxemia and causes cell and mitochondrial oxygen utilization disorders (Murray 2016). The hypobaric hypoxic environment tends to increase the severity of inflammation, which can lead to brain damage (Li et al. 2017), pulmonary edema, and multiple organ dysfunction syndrome (MODS) which is the most complicated and difficult to cure (Swank \& Deitch 1996).

When entering high-altitude areas residents living at low altitudes will experience physiological reactions including changes in respiration and cardiovascular function, erythropoiesis factors, and gastrointestinal symptoms. The mechanism of the gastrointestinal symptoms of acute mountain sickness (AMS) caused by rising to high altitude is unclear (Fruehauf et al. 2020). 
56 Individuals with sufficient adaptability to high-altitude can maintain homeostasis under hypoxic

57

58

59

60

61

62

63

64

65

66

67

68

69

70

71

conditions, but the metabolism and physiological functions will be affected, such as changes in energy metabolism in the heart and skeletal muscles. In other studies, the levels of Adenosine Triphosphate (ATP) and phosphocreatine (PCr) in skeletal muscle decreased with the altitude increases and this decrease continued over time. This indicates that decreasing ATP demand in high-altitude areas cannot meet the requirement of suppressing ATP supply (Hochachka et al. 1996).

In recent years, while excluding the symptoms of AMS, the incidence of gastrointestinal symptoms is extremely high, mainly manifested as abdominal pain, diarrhea, nausea, vomiting, and even hematochezia and melena for symptoms of gastrointestinal bleeding (Frühauf 2014). Studies have shown that gastrointestinal mucosal barrier function damage caused by severe trauma, infection, hemorrhagic shock, and other factors can activate the inflammatory response pathway (Dempsey et al. 2019). The intestine has essential biological functions at different oxygen concentrations (Sengupta et al. 2014). Due to the existence of numerous gut microbiome, the intestine is in a hypoxic environment which maintains nutrient absorption, immune defense, and intestinal barrier functions (Singhal \& Shah 2020).

At present, various studies have shown that oxygen metabolism is closely related to the intestinal homeostasis environment and the occurrence of many intestinal diseases is a result of the destruction of oxygen gradients (Ramakrishnan \& Shah 2016). Under hypoxic conditions, cells adapt to the hypoxic environment by adjusting gene expression. Many metabolic changes are regulated by hypoxia inducible factors (HIFs). The encoding of enzymes regulated by HIFs involves glycolytic metabolism, lipid metabolism, nutrient absorption, peroxisomal metabolism, and mitochondrial function (Xie \& Simon 2017). Furthermore, it has been shown that hypoxia can increase leukocyte adhesion, stimulate mucosal tissues inflammation, and destroy the tissue barrier function (Deng et al. 2018; Saeedi et al. 2015; Seys et al. 2013). Regulating HIFs promote the expression of transcription factors in hypobaric conditions and through hypoxia, but how HIFs are regulated in hypobaric hypoxia and the downstream response is not clear (Wang et al. 2018). We conducted a more in-depth study through RNA-Seq analysis to explore the effects of 2 to 4 weeks of hypobaric hypoxic conditions on intestinal gene expression in in vivo studies.

\section{Materials \& Methods}

\section{Animals and treatment}

Adult male Sprague Dawley (SD) rats were randomly divided into 3 groups with 4 rats in each group, but during modeling both the $\mathrm{S}$ and W2S groups lost one rat. According to the study by Qian Ni et al. (2014), we divided the experimental rats into three groups: the normal control group (S), the acute hypobaric hypoxia group (W2S) which was exposed to hypobaric hypoxic conditions for 2 weeks, and the chronic hypobaric hypoxia group (W4S) which was exposed to hypobaric hypoxic conditions for 4 weeks. The hypobaric hypoxic environment was constructed

Peer) reviewing PDF | (2020:11:55568:1:2:NEW 2 Jun 2021) 
93 to simulate a 5,500-meter-high atmospheric environment using a FLYDWC50-1C hypobaric

94 hypoxia cabin (Guizhou Fenglei Air Ordnance LTD, Guizhou, China). The rats were

95 anesthetized with $10 \%$ chloral hydrate $(0.4 \mathrm{ml} / 100 \mathrm{~g})$, the small intestine tissue was taken out,

96 washed quickly, and stored in liquid nitrogen. The study was approved by the Animal Ethics

97 Committee of the Chinese PLA General Hospital (2017-X13-05).

98 RNA extraction

99 The small intestine sample was chopped and mixed with liquid nitrogen to form a powder. Add

100 Trizol reagent to dissociate nucleoprotein; add $0.3 \mathrm{ml}$ chloroform, shake for 15 seconds, and wait

101 at room temperature for 2 minutes; $12000 \mathrm{rpm}$ centrifuge $\left(4^{\circ} \mathrm{C}\right.$ for 15 minutes), add $0.5 \mathrm{ml}$

102 isopropanol and wait for 15 minutes at room temperature; $12000 \mathrm{rpm}\left(4^{\circ} \mathrm{C}\right.$ for 15 minutes $)$,

103 aspirate the supernatant; add $1 \mathrm{~mL} 4^{\circ} \mathrm{C}$ pre-cooled $75 \%$ ethanol to the RNA at the bottom;

104 centrifuge $\left(10,000 \mathrm{rpm}, 5\right.$ minutes, $\left.4^{\circ} \mathrm{C}\right)$, and aspirate the supernatant; centrifuge $(10,000 \mathrm{rpm}$,

$1054^{\circ} \mathrm{C}, 5$ minutes), aspirate the remaining liquid, and ventilate for 10 minutes; RNA concentration

106 was determined in a Qubit $\mathbb{Q} 2.0$ Fluorometer (Life Technologies, Carlsbad, CA, USA) following

107 the Qubit $\mathbb{Q}$ RNA Assay Kit operating instruction.

108 Transcriptome library construction and Sequence

109 Reverse transcription synthesis of the first strand cDNA, recombination into the second strand of

110 DNA; then according to the above template in vitro transcription synthesis of cDNA, cDNA

111 purification and reverse recording, purification, and quantification of the reverse transcription

112 cDNA, and finally the library quality was assessed. The small intestine samples were sequenced

113 with TruSeq PE Cluster Kit v3-cBot-HS (Illumina). All analyses are based on clean data.

\section{Reference genome alignment}

115 Reference genome was obtained from the relevant genome analysis website. The required gene

116 annotation files were taken from the database as well. Using the HISAT2 v 2.0.4 comparison tool,

117 the paired end readings were aligned with the reference genome (Langmead \& Salzberg 2012).

\section{Differential expression analysis}

119 The analysis of differentially expressed genes (DEGs) was finished through the Limma package

120 in R language. Differentially expressed genes are based on the standard of $|\operatorname{logFC}|>1, \mathrm{P}$

121 value $<0.05$. Genes with $\operatorname{logFC}>1$ are defined as an up-regulated gene. Genes with $\operatorname{LogFC}<-1$

122 are defined as a down-regulated gene.

\section{Functional analysis and co-expression analysis}

124 Gene ontology (GO) was implemented using the DEGs in three functional categories: biological

125 process (BP), molecular function (MF), and cellular component (CC). The Kyoto Encyclopedia

126 of Genes and Genomes (KEGG) pathway database was used to annotate DEGs on the pathway in 
127 the database for statistical analysis (Mao et al. 2005). The co-expression gene set analysis was

128 finished using the Cogena software package (Jia et al. 2016).

129

\section{qRT-PCR analysis}

131 Total RNA was collected using the TRIzol reagent (TaKaRa, Japan) following the manufacturer's instructions. Then RNA was reverse transcribed to cDNA using reverse transcriptase (Promega GoTaq qPCR Master Mix, China). qRT-PCR analysis was actualized by Bio-Rad CFX96 Real-Time PCR Detection System (Bio-Rad, U.S.).

\section{Results}

\section{Differential gene expression analysis}

137 Through differential gene expression analysis, we found that these three groups of samples had different responses to the hypobaric hypoxic environment. 636 differentially expressed genes were screened. These DEGs mainly involved ATP-binding cassette (Abc), ELOVL fatty acid elongase 2 (Elov12), isocitrate dehydrogenase $\mathrm{NADP}(+) 1$ (Idh1), apolipoprotein $\mathrm{B}$ (Apob), lipase C (Lipc), perilipin 1/2 (Plin1/ Plin2), fatty acid binding protein (Fabp), solute carrier family (Slc), angiotensin I converting enzyme 2 (Ace2), and membrane metallo-endopeptidase (Mme). The functions of these genes are associated with ATPase activity, fatty acid metabolic process, NADP metabolic process, cholesterol and lipid transport, thiamine (vitamin B) transmembrane transporter activity, and the renin-angiotensin system. The hierarchical clustering of the DEGs is shown on the heat map in Figure 1. W2S represented acute hypobaric hypoxia, while the W4S group represented chronic hypobaric hypoxia. The expression patterns of DEGs were vastly different from each other. After analysis, we found that the relationship between the W4S and the S groups were more closely related when compared with the W2S groups.

151

There are 195/255 up-regulated genes in the W2S/W4S groups, respectively, compared with the $\mathrm{S}$ group. There are 120 up-regulated genes and 52 down-regulated genes in both the W2S and W4S groups compared with the S group and 4 genes up-regulated in the W2S but downregulated in the W4S. Conversely, there are 19 genes down-regulated in the W2S but upregulated in the W4S (Figure 2, Table 1). Many of the up-regulated genes both in the W2S and the W4S groups, such as Tpsab1, Slc19a3, and LOC103694855, are associated with identical protein binding, transmembrane transport, and oxygen carrier activity. These up-regulated gene expressions may be associated with hypobaric hypoxia.

Further principal component analyses suggested similar results as the observation above (Figure 3). The three groups are separated, but the members in the same group are clustered together; this indicated that the model construction was successful. 
162 Biological functional analysis of DEGs

163 To explore the impact of hypobaric hypoxia on gene function, we used Gene Ontology (GO)

164 analysis. DEGs are classified into three functional categories. For biological processes, the GO

165 analysis indicated that nutrient transport ontologies, especially anion, fatty, and acid were

166 enriched. For molecular function, some transmembrane transporter ontologies, such as metal ion

167 and active, were enriched. For cellular components, the DEGs were active in the plasma

168 membrane (Figure 4).

169 We used KEGG pathway enrichment analysis to further explore the biological functions of

170 DEGs in response to hypobaric hypoxia. Several metabolic and digestive pathways, such as

171 PPAR signaling pathway, glycerolipid metabolism, fat metabolism, mineral absorption,

172 ferroptosis, and vitamin metabolism were identified (Figure 5).

\section{Co-expression analysis}

174 Co-expression analysis can identify genes with similar functions; in this co-expression analysis,

175 we observed three patterns. Most of the genes in the first cluster were down-regulated in the $2^{\text {nd }}$

176 week and up-regulated in the $4^{\text {th }}$ week. In the second cluster, 126 genes were down-regulated

177 until the $4^{\text {th }}$ week. In the third cluster, 244 genes were up-regulated from the $2^{\text {nd }}$ week until the

$1784^{\text {th }}$ week (Figure 6).

179 The Cogena Bioconductor software package was used for pathway analysis of each co-

180 expression cluster. According to the manual, we selected three clusters for analysis and used the

181 k-means clustering method. Interestingly, metabolic and digestive related pathways, such as

182 PPAR and the renin angiotensin pathway, and fatty acid metabolism, are only enriched in the

183 third cluster (Figure 7). In the third cluster, we found that the metabolic and digestive pathways

184 start to work in the $2^{\text {nd }}$ week.

185

186

187

188

189

190

191

\section{Gene transcription}

The most changed genes, including the top 20 genes with increased expression and top 20 with decreased expression, have been listed (Figure 8). The top 5 differentially expressed genes for each group, including the up-regulated genes and down-regulated genes, were detected through RT-PCR (Figure 9). The trend of genes differentially expressed between each group is mostly matched with the transcriptional landscape.

\section{Discussion}

193 The gastrointestinal tract is the key organ involved in the digestion of food and providing 194 nutrients to the body for proper maintenance. However, the hypobaric hypoxic environment of 195 the medical plateau can cause dysfunction of the gastrointestinal system. A previous study found 196 that hypobaric hypoxia can effectively regulate immune/inflammatory processes and energy 197 metabolism (Gangwar et al. 2020), and the degree of change has a clear relationship with time 198 (Padhy et al. 2016). The high-altitude hypoxic environment destroys lipid metabolism and 
199 activates inflammatory processes, which causes abnormal metabolic function. Related studies

200 have shown that the liver X receptor (LXR) plays an essential role in cholesterol, fatty acid, and

201 glucose metabolism (Li \& Glass 2004). The physiological functions of LXRs have a close

202 relationship with nuclear receptors (such as PPAR) (Hong \& Tontonoz 2014). The main role of

203 the LXR pathway is based on energy homeostasis of lipid metabolism. In the high-altitude

204 hypoxia model, the LXR pathway is strongly activated in the later stage (Tang et al. 2014).

205 However, its mechanism of action in a hypoxic environment is still unclear, and the changes in

206 the pathway may affect lipid metabolism. Several groups are currently conducting drug research

207 and development based on LXR. Some studies have found that LXR activates macrophages

208 through stearoyl-CoA desaturase (Wang et al. 2004). The latest findings indicate that

209 inflammation regulation is closely related to lipid metabolism. This study provides the first basis

210 for investigating the changes in transcription and signaling pathways in rat small intestine tissues

211 under acute and chronic hypobaric hypoxic conditions and found that the hypobaric hypotension

212 environment affects fatty acid metabolism in the small intestine. These findings indicate that

213 metabolic and digestive related pathways play a key role in the differences in the mechanism

214 between hypobaric hypoxia and acute/chronic mountain sickness.

215 Studies have shown that oxygen metabolism is closely related to the intestinal homeostasis

216 environment (Ramakrishnan \& Shah 2016). Under hypoxic conditions, many metabolic changes

217 are regulated by hypoxia inducible factors (HIFs). The encoding of enzymes regulated by HIFs

218 involves glycolytic metabolism, lipid metabolism, and nutrient absorption (Xie \& Simon 2017).

219 Hypoxia can increase leukocyte adhesion, stimulate mucosal tissues inflammation, and destroy

220 the tissue barrier function (Deng et al. 2018; Saeedi et al. 2015; Seys et al. 2013). By studying

221 the changes of gene expression in small intestine tissues exposed to hypobaric hypoxia for 2 to 4

222 weeks, these results provide a basis for further research on the molecular pathogenesis of

223 diseases under hypobaric hypoxic conditions. Through genome-wide transcription analysis, we

224 found that hypoxia increased pro-inflammatory cytokines release, causing damage to the small

225 intestine metabolism from the $2^{\text {nd }}$ week. This study plays an important part in the research of the

226 molecular mechanism of small intestine injury in high altitude areas and sets the stage for further

227 genetic and functional research. There is a clear correlation between systemic inflammation and

228 AMS (Boos et al. 2016; Li et al. 2015). A likely mechanism is that hypoxia changes cellular

229 immunity and regulates the release of cytokines. Studies have shown that intermittent hypoxia

230 has links with metabolic dysfunction (including abnormal lipid metabolism and insulin

231 resistance). Intermittent hypoxia may induce sympathetic activation, increased inflammation,

232 regulate hormone metabolism, and directly cause pancreatic $\beta$-cell damage to interfere with

233 glucose metabolism (Drager et al. 2010). At the same time, in another study, scholars found that

234 intermittent hypoxia conditions can increase the rate of lipid oxidation and suppress carbohydrate

235 oxidation (Kelly \& Basset 2017). Previous reports have indicated that this severe intermittent

236 hypoxia brings about a series of changes, such as low-grade inflammation, oxidative stress, and

237 endoplasmic reticulum stress (Chacaroun et al. 2020). These adverse reactions are harmful to 
238 many systems, such as cardiovascular, respiratory, and metabolism, as well as cognition etc.

239 (Chacaroun et al. 2020; Gabryelska et al. 2020; Gauda et al. 2020; Vermeulen et al. 2020).

240 In a previous study, Sweet $\mathrm{R}$ et al found that hypoxia can up-regulate the expression of S100a8,

241 the Retn gene, and Slc19a3 (Jiao et al. 2019; Sweet et al. 2010; Uchiyama et al. 2019). The

242 transcriptional landscape and RT-PCR results of this study align with the trends of other

243 researchers, indicating that this study will be a useful reference for future studies on intestinal

244 diseases under hypobaric hypoxia. The angiotensin I converting enzyme 2 (Ace2) gene, which

245 regulates systemic arterial blood pressure through the renin-angiotensin system, and the

246 membrane metallo-endopeptidase (Mme) gene, that influences kidney development, are the

247 DEGs found in this study, they play an important role in renin-angiotensin system and metabolic

248 disorders related to the renin-angiotensin pathway were also observed. Revera $\mathrm{M}$ et al. found that

249 during acute and chronic exposure to high altitude, the aortic pulse wave velocity increased

250 significantly with altitude and the subendocardial oxygen supply/demand index significantly

251 decreased (Revera et al. 2017). Acute exposure to hypobaric hypoxia can cause aortic stiffness

252 and reduce subendocardial blood supply. A randomized clinical trial found that as the altitude

253 increases, blood pressure will gradually increase, and will remain elevated for 3 weeks after

254 entering the high-altitude area. Angiotensin receptor blockers can reduce blood pressure at 3400

255 meters, but the effect is not apparent at 5400 meters (Parati et al. 2014). A significant feature of

256 hypoxic pulmonary hypertension is the remodeling of the pulmonary artery (Mikami et al. 1996).

257 In another study by Mikami O et al. 1996, it was found that the angiotensin II (Ang II) receptors

258 increased in the lung tissue of rats with hypoxic pulmonary hypertension, the Ang II level rose,

259 and the renin-angiotensin system activated. Then, it induced hypertrophy and proliferation of

260 aortic smooth muscle cells and cardiomyocytes (Parati et al. 2014).

261 Our study found that mineral absorption is overexpressed in a hypobaric hypoxic environment.

262 Hypoxic environments and HIFs play an important role in the absorption of iron and minerals in

263 the small intestine (Das et al. 2015; Das et al. 2020). The absorption of bones is affected by the

264 hypobaric hypoxic environment (Guner et al. 2013) which causes abnormal absorption of

265 minerals. This indicates that the hypobaric hypoxic environment will interfere with the

266 metabolism of rats. This metabolic change may be the body's adaptation to a hypobaric hypoxic

267 environment.

268 This study has a few limitations including an insufficient number of subjects, which generated an

269 accurate cut-off value for the diagnosis of AMS in rats. Also, since no blood samples were

270 obtained in this experiment, we were unable to investigate plasma-related indicators.

\section{Conclusion}

272 This study found 636 DEGs associated with ATPase activity, fatty acid metabolic process,

273 NADP metabolic process, cholesterol and lipid transport, thiamine (vitamin B) transmembrane

274 transporter activity, and the renin-angiotensin system. KEGG pathway analysis found that in

275 hypobaric hypoxic environments metabolic and digestive pathways, such as PPAR signaling 
276

277

278

279

280

281

282

283

284

285

286

287

288

289

290

291

292

293

294

295

296

297

298

299

300

301

302

303

304

305

306

307

308

309

310

311

312

313

pathway, mineral absorption, glycerolipid metabolism, fat digestion and absorption, ferroptosis, vitamin digestion and absorption, are highly enriched. Gene Ontology analysis revealed similar results. Cogena analysis discovered that up-regulation of digestive and metabolic functions began from the $2^{\text {nd }}$ week of high-altitude exposure. This research provides new information regarding the molecular effects of the hypobaric hypoxic environment and raises new questions about lipid metabolism disorders, inflammation, and redox stress. Further study of the relationship between them is needed to explore the potential molecular mechanism of gastrointestinal regulation under hypobaric hypoxic environment.

\section{References}

Boos CJ, Woods DR, Varias A, Biscocho S, Heseltine P, and Mellor AJ. 2016. High Altitude and Acute Mountain Sickness and Changes in Circulating Endothelin-1, Interleukin-6, and Interleukin-17a. High Alt Med Biol 17:25-31. 10.1089/ham.2015.0098

Chacaroun S, Borowik A, Doutreleau S, Belaidi E, Wuyam B, Tamisier R, Pépin JL, Flore P, and Verges S. 2020. Cardiovascular and metabolic responses to passive hypoxic conditioning in overweight and mildly obese individuals. Am J Physiol Regul Integr Comp Physiol 319:R211-R222. 10.1152/ajpregu.00311.2019

Das N, Xie L, Ramakrishnan SK, Campbell A, Rivella S, and Shah YM. 2015. Intestine-specific Disruption of Hypoxia-inducible Factor (HIF)-2 $\alpha$ Improves Anemia in Sickle Cell Disease. J Biol Chem 290:23523-23527. 10.1074/jbc.C115.681643

Das NK, Schwartz AJ, Barthel G, Inohara N, Liu Q, Sankar A, Hill DR, Ma X, Lamberg O, Schnizlein MK, Arqués JL, Spence JR, Nunez G, Patterson AD, Sun D, Young VB, and Shah YM. 2020. Microbial Metabolite Signaling Is Required for Systemic Iron Homeostasis. Cell Metab 31:115-130.e116. 10.1016/j.cmet.2019.10.005

Dempsey JL, Little M, and Cui JY. 2019. Gut microbiome: An intermediary to neurotoxicity. Neurotoxicology 75:41-69. 10.1016/j.neuro.2019.08.005

Deng Y, Chen G, Zhou R, Wu W, You Z, Meng W, Yang L, Qiu Y, Liu J, and Li T. 2018. Direct evidence that hypoxia triggers the cardioprotective response of ischemic preconditioning in a dog double-circuit cardiopulmonary bypass model. Life Sci 209:395-402. 10.1016/j.1fs.2018.08.042

Drager LF, Jun JC, and Polotsky VY. 2010. Metabolic consequences of intermittent hypoxia: relevance to obstructive sleep apnea. Best Pract Res Clin Endocrinol Metab 24:843-851. 10.1016/j.beem.2010.08.011

Fruehauf H, Vavricka SR, Lutz TA, Gassmann M, Wojtal KA, Erb A, Maggiorini M, Schwizer W, Fried M, Fox M, Goetze O, and Greuter T. 2020. Evaluation of Acute Mountain Sickness by Unsedated Transnasal Esophagogastroduodenoscopy at High Altitude. Clin Gastroenterol Hepatol 18:2218-2225.e2212. 10.1016/j.cgh.2019.11.036

Frühauf H. 2014. [Every mountain too high when Nausea strikes: gastrointestinal function at high altitude]. Praxis (Bern 1994) 103:825-832. 10.1024/1661-8157/a001718 
314 Gabryelska A, Karuga FF, Szmyd B, and Białasiewicz P. 2020. HIF-1 $\alpha$ as a Mediator of Insulin

315

316

317

318

319

320

321

322

323

324

325

326

327

328

329

330

331

332

333

334

335

336

337

338

339

340

341

342

343

344

345

346

347

348

349

350

351

352

Resistance, T2DM, and Its Complications: Potential Links With Obstructive Sleep Apnea.

Front Physiol 11:1035. 10.3389/fphys.2020.01035

Gangwar A, Paul S, Ahmad Y, and Bhargava K. 2020. Intermittent hypoxia modulates redox homeostasis, lipid metabolism associated inflammatory processes and redox posttranslational modifications: Benefits at high altitude. Sci Rep 10:7899. 10.1038/s41598020-64848-X

Gauda EB, Conde S, Bassi M, Zoccal DB, Almeida Colombari DS, Colombari E, and Despotovic N. 2020. Leptin: Master Regulator of Biological Functions that Affects Breathing. Compr Physiol 10:1047-1083. 10.1002/cphy.c190031

Guner I, Uzun DD, Yaman MO, Genc H, Gelisgen R, Korkmaz GG, Hallac M, Yelmen N, Sahin G, Karter Y, and Simsek G. 2013. The effect of chronic long-term intermittent hypobaric hypoxia on bone mineral density in rats: role of nitric oxide. Biol Trace Elem Res 154:262-267. 10.1007/s12011-013-9722-8

Hochachka PW, Buck LT, Doll CJ, and Land SC. 1996. Unifying theory of hypoxia tolerance: molecular/metabolic defense and rescue mechanisms for surviving oxygen lack. Proc Natl Acad Sci U S A 93:9493-9498. 10.1073/pnas.93.18.9493

Hong C, and Tontonoz P. 2014. Liver X receptors in lipid metabolism: opportunities for drug discovery. Nat Rev Drug Discov 13:433-444. 10.1038/nrd4280

Jia Z, Liu Y, Guan N, Bo X, Luo Z, and Barnes MR. 2016. Cogena, a novel tool for coexpressed gene-set enrichment analysis, applied to drug repositioning and drug mode of action discovery. BMC Genomics 17:414. 10.1186/s12864-016-2737-8

Jiao J, Wang C, and Zhang L. 2019. Epithelial physical barrier defects in chronic rhinosinusitis. Expert Rev Clin Immunol 15:679-688.10.1080/1744666X.2019.1601556

Kelly LP, and Basset FA. 2017. Acute Normobaric Hypoxia Increases Post-exercise Lipid Oxidation in Healthy Males. Front Physiol 8:293. 10.3389/fphys.2017.00293

Langmead B, and Salzberg SL. 2012. Fast gapped-read alignment with Bowtie 2. Nat Methods 9:357-359. 10.1038/nmeth.1923

Li AC, and Glass CK. 2004. PPAR- and LXR-dependent pathways controlling lipid metabolism and the development of atherosclerosis. J Lipid Res 45:2161-2173. 10.1194/j1r.R400010JLR200

Li D, Zhang L, Huang X, Liu L, He Y, Xu L, Zhang Y, Zhao T, Wu L, Zhao Y, Wu K, Wu Y, Fan M, and Zhu L. 2017. WIP1 Phosphatase Plays a Critical Neuroprotective Role in Brain Injury Induced by High-Altitude Hypoxic Inflammation. Neurosci Bull 33:292-298. 10.1007/s12264-016-0095-9

Li G, Fu J, Zhao Y, Ji K, Luan T, and Zang B. 2015. Alpha-lipoic acid exerts anti-inflammatory effects on lipopolysaccharide-stimulated rat mesangial cells via inhibition of nuclear factor kappa B (NF-אB) signaling pathway. Inflammation 38:510-519. 10.1007/s 10753014-9957-3

Peer) reviewing PDF | (2020:11:55568:1:2:NEW 2 Jun 2021) 
353 Mao X, Cai T, Olyarchuk JG, and Wei L. 2005. Automated genome annotation and pathway

354

355

356

357

358

359

360

361

362

363

364

365

366

367

368

369

370

371

372

373

374

375

376

377

378

379

380

381

382

383

384

385

386

387

388

389

390

391 identification using the KEGG Orthology (KO) as a controlled vocabulary. Bioinformatics 21:3787-3793. 10.1093/bioinformatics/bti430

Mikami O, Nagaoka I, Hasunuma K, and Takahashi H. 1996. [Angiotensin II receptors in a rat model of hypobaric hypoxic pulmonary hypertension]. Nihon Kyobu Shikkan Gakkai Zasshi 34:186-193.

Murray AJ. 2016. Energy metabolism and the high-altitude environment. Exp Physiol 101:23-27. 10.1113/EP085317

Ni Q, Shao Y, Wang YZ, Jing YH, and Zhang YC. 2014. Impact of high altitude on the hepatic fatty acid oxidation and synthesis in rats. Biochem Biophys Res Commun 446:574-579. 10.1016/j.bbrc.2014.03.001

Padhy G, Gangwar A, Sharma M, Himashree G, Singh K, Bhaumik G, Bhargava K, and Sethy NK. 2016. Plasma kallikrein-bradykinin pathway promotes circulatory nitric oxide metabolite availability during hypoxia. Nitric Oxide 55-56:36-44. 10.1016/j.niox.2016.02.009

Parati G, Bilo G, Faini A, Bilo B, Revera M, Giuliano A, Lombardi C, Caldara G, Gregorini F, Styczkiewicz K, Zambon A, Piperno A, Modesti PA, Agostoni P, and Mancia G. 2014. Changes in $24 \mathrm{~h}$ ambulatory blood pressure and effects of angiotensin II receptor blockade during acute and prolonged high-altitude exposure: a randomized clinical trial. Eur Heart J 35:3113-3122. 10.1093/eurheartj/ehu275

Ramakrishnan SK, and Shah YM. 2016. Role of Intestinal HIF-2 $\alpha$ in Health and Disease. Annu Rev Physiol 78:301-325. 10.1146/annurev-physiol-021115-105202

Revera M, Salvi P, Faini A, Giuliano A, Gregorini F, Bilo G, Lombardi C, Mancia G, Agostoni P, Parati G, and Investigators HH. 2017. Renin-Angiotensin-Aldosterone System Is Not Involved in the Arterial Stiffening Induced by Acute and Prolonged Exposure to High Altitude. Hypertension 70:75-84. 10.1161/HYPERTENSIONAHA.117.09197

Saeedi BJ, Kao DJ, Kitzenberg DA, Dobrinskikh E, Schwisow KD, Masterson JC, Kendrick AA, Kelly CJ, Bayless AJ, Kominsky DJ, Campbell EL, Kuhn KA, Furuta GT, Colgan SP, and Glover LE. 2015. HIF-dependent regulation of claudin-1 is central to intestinal epithelial tight junction integrity. Mol Biol Cell 26:2252-2262. 10.1091/mbc.E14-071194

Sengupta C, Ray S, and Chowdhury R. 2014. Fine tuning of virulence regulatory pathways in enteric bacteria in response to varying bile and oxygen concentrations in the gastrointestinal tract. Gut Pathog 6:38. 10.1186/s13099-014-0038-9

Seys SF, Daenen M, Dilissen E, Van Thienen R, Bullens DM, Hespel P, and Dupont LJ. 2013. Effects of high altitude and cold air exposure on airway inflammation in patients with asthma. Thorax 68:906-913. 10.1136/thoraxjnl-2013-203280

Singhal R, and Shah YM. 2020. Oxygen battle in the gut: Hypoxia and hypoxia-inducible factors in metabolic and inflammatory responses in the intestine. J Biol Chem 295:10493-10505. 
392

393

394

395

396

397

398

399

400

401

402

403

404

405

406

407

408

409

410

411

412

413

414

415

416

417

418

419

10.1074/jbc.REV120.011188

Swank GM, and Deitch EA. 1996. Role of the gut in multiple organ failure: bacterial translocation and permeability changes. World J Surg 20:411-417. 10.1007/s002689900065

Sweet R, Paul A, and Zastre J. 2010. Hypoxia induced upregulation and function of the thiamine transporter, SLC19A3 in a breast cancer cell line. Cancer Biol Ther 10:1101-1111. 10.4161/cbt.10.11.13444

Taino G, Giardini G, Delogu A, Foti R, Oddone E, and Imbriani M. 2019. [Work on a building site at high altitude: physiopathological features and entailments for the occupational medicine]. G Ital Med Lav Ergon 41:242-252.

Tang H, Mirshahidi S, Senthil M, Kazanjian K, Chen CS, and Zhang K. 2014. Down-regulation of LXR/RXR activation and negative acute phase response pathways in colon adenocarcinoma revealed by proteomics and bioinformatics analysis. Cancer Biomark 14:313-324. 10.3233/CBM-140409

Uchiyama T, Itaya-Hironaka A, Yamauchi A, Makino M, Sakuramoto-Tsuchida S, Shobatake R, Ota H, Takeda M, Ohbayashi C, and Takasawa S. 2019. Intermittent Hypoxia UpRegulates. Int J Mol Sci 20. 10.3390/ijms20081960

Vermeulen TD, Benbaruj J, Brown CV, Shafer BM, Floras JS, and Foster GE. 2020. Acute intermittent hypercapnic hypoxia and cerebral neurovascular coupling in males and females. Exp Neurol 334:113441. 10.1016/j.expneurol.2020.113441

Wang C, Jiang H, Duan J, Chen J, Wang Q, and Liu X. 2018. Exploration of Acute Phase Proteins and Inflammatory Cytokines in Early Stage Diagnosis of Acute Mountain Sickness. High Alt Med Biol 19:170-177. 10.1089/ham.2017.0126

Wang Y, Kurdi-Haidar B, and Oram JF. 2004. LXR-mediated activation of macrophage stearoylCoA desaturase generates unsaturated fatty acids that destabilize ABCA1. J Lipid Res 45:972-980. 10.1194/jlr.M400011-JLR200

Xie H, and Simon MC. 2017. Oxygen availability and metabolic reprogramming in cancer. $J$ Biol Chem 292:16825-16832. 10.1074/jbc.R117.799973

Peer) reviewing PDF | (2020:11:55568:1:2:NEW 2 Jun 2021) 
Figure 1

Heatmap of the DEGs.

There are 636 DEGs were found in the three groups. The red color represents the high expression of the genes, while the blue represents low expression of the genes.

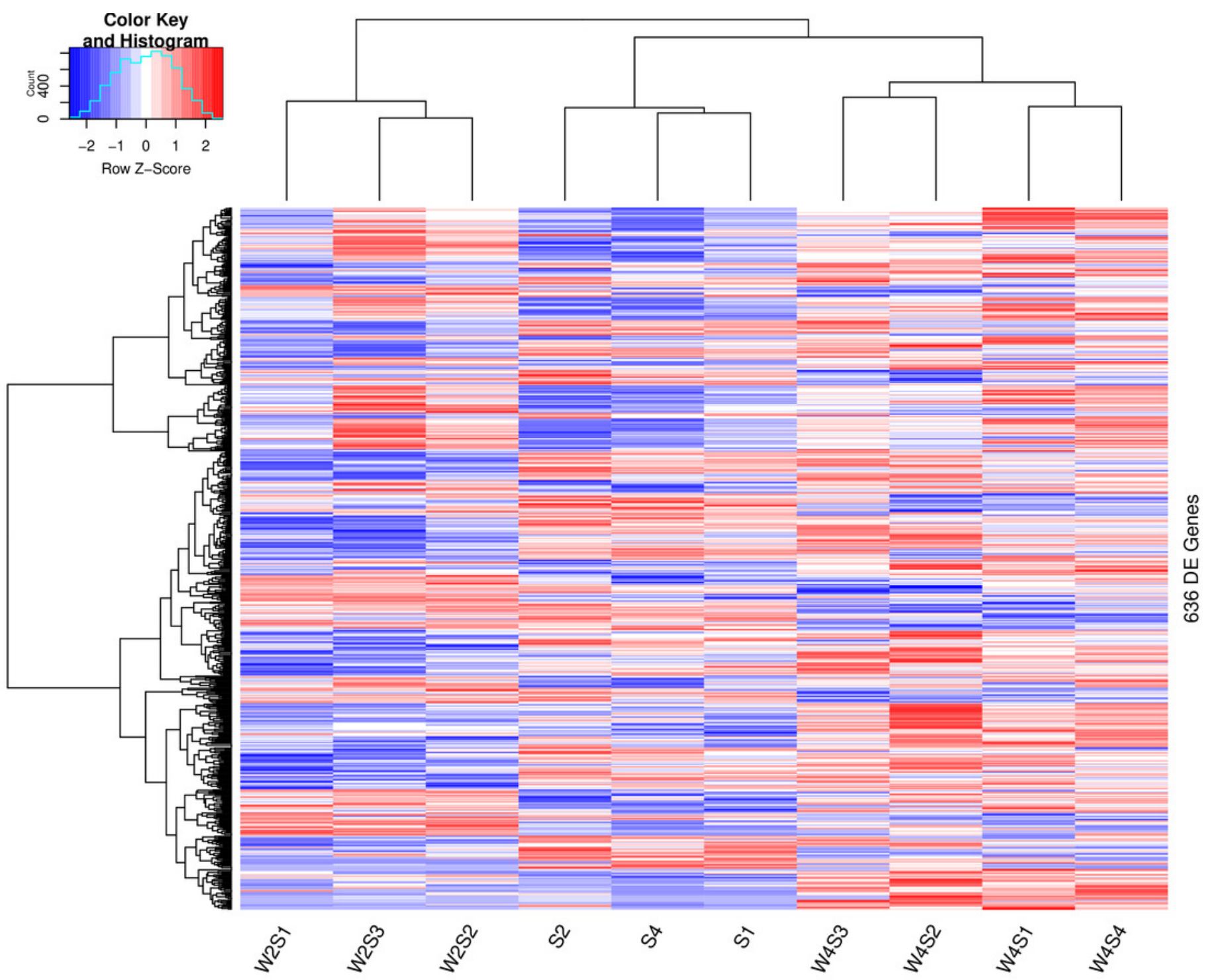




\section{Figure 2}

Differential gene expression in W2S and W4S

Genes with $\log F C>1$ are defined as an up-regulated gene. Genes with LogFC $<-1$ is defined as a down-regulated gene. There are 195/ 255genes up-regulated in W2S/W4S respectively compared with S. The genes up-regulated both in W2S and W4S groups are 120, and downregulated both in two groups are 52 compared with S group. There are 4 genes up-regulated in W2S but down-regulated in W4S. Conversely, There are 19 gens down-regulated in W2S but up-regulated in W4S. 


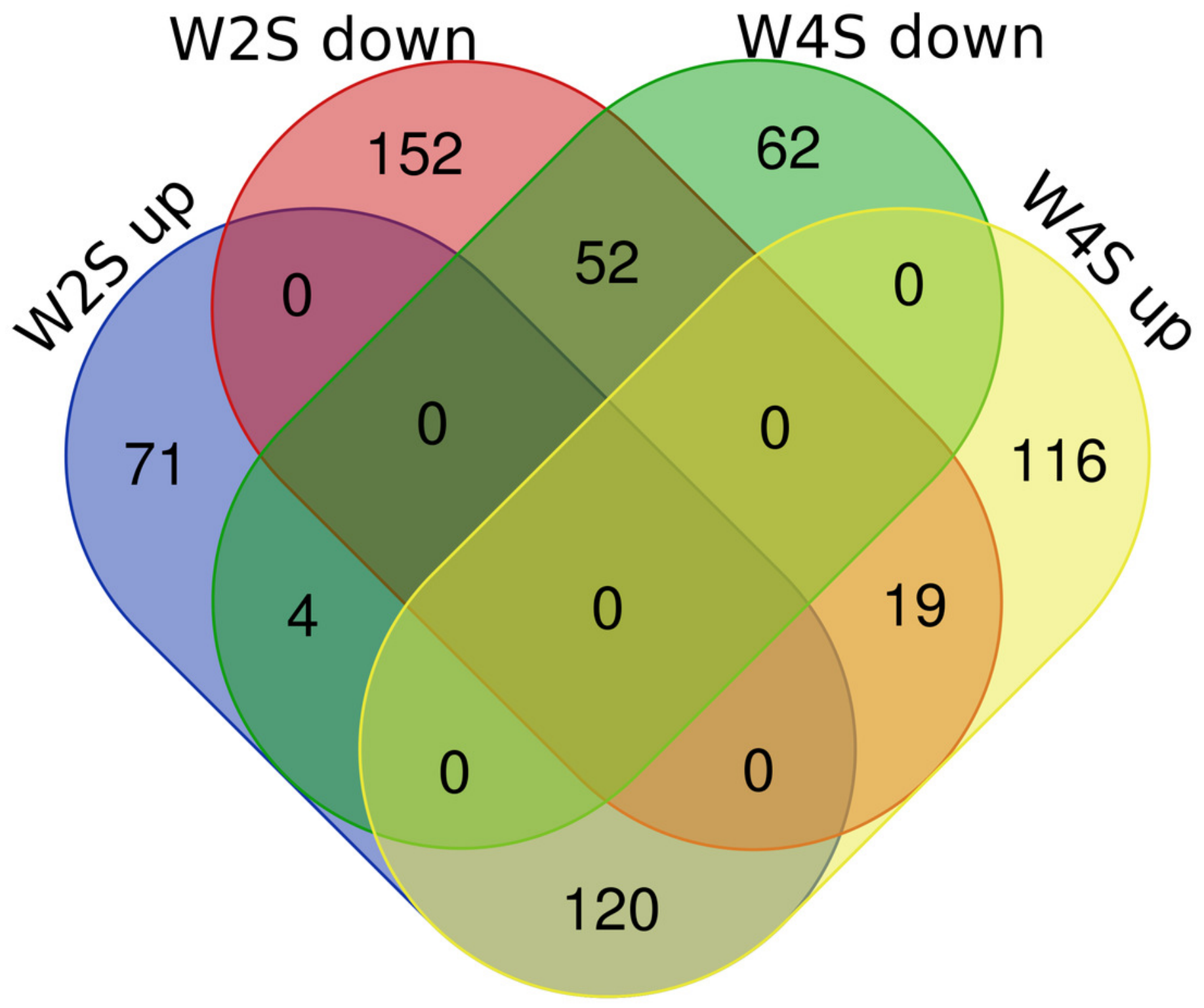


Figure 3

Principal component analysis of the samples in the three groups.

All of these three groups are separated from each other, but the members in the same group are clustered together.

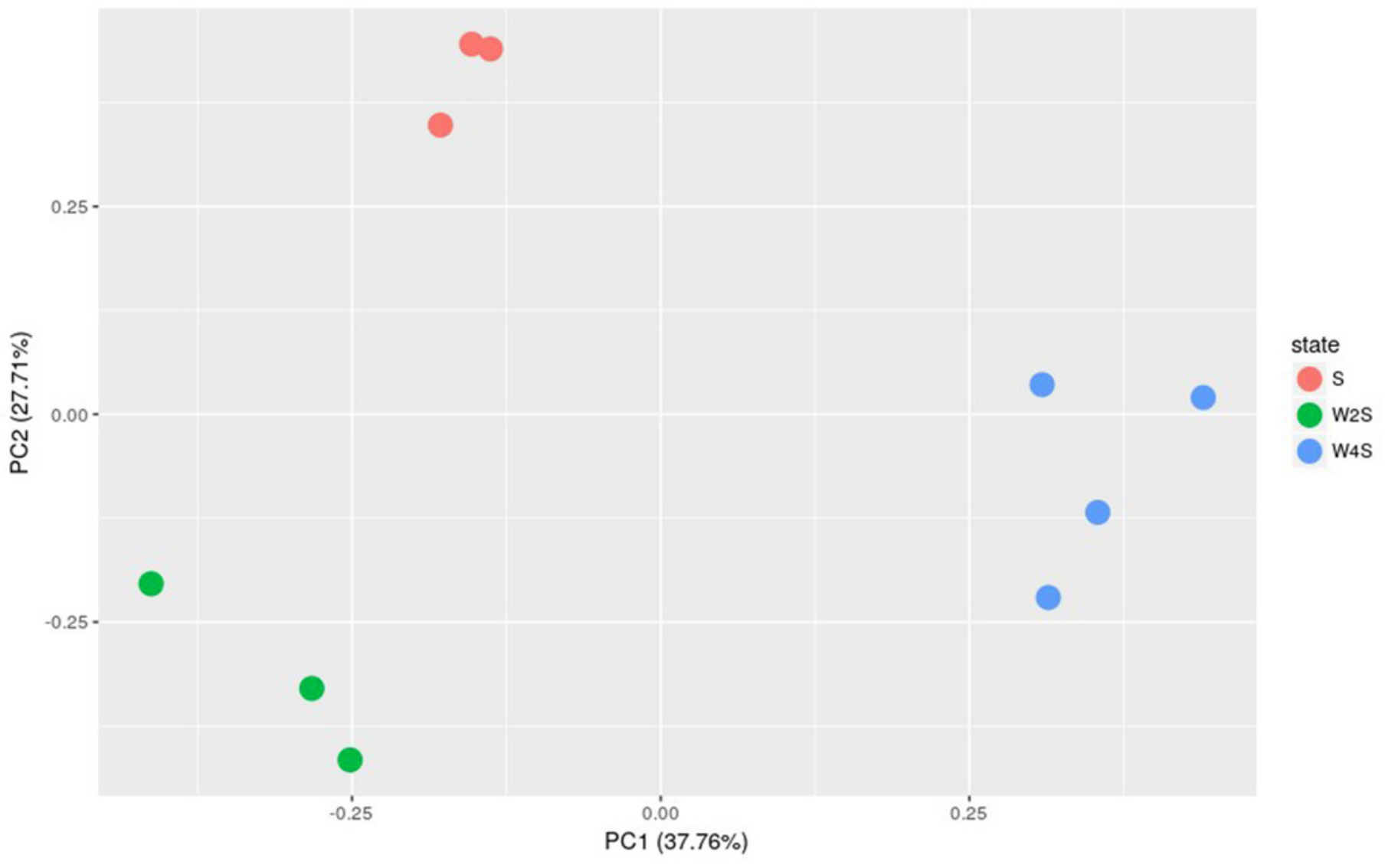


Figure 4

GO analysis of the DEGs.

(A) biological process. (B) molecular function. (C) cellular component. The Y-axis is the subordinate GO term of the three major categories of GO, and the x-axis is the number of genes under the term and the percentage of the total number of genes.
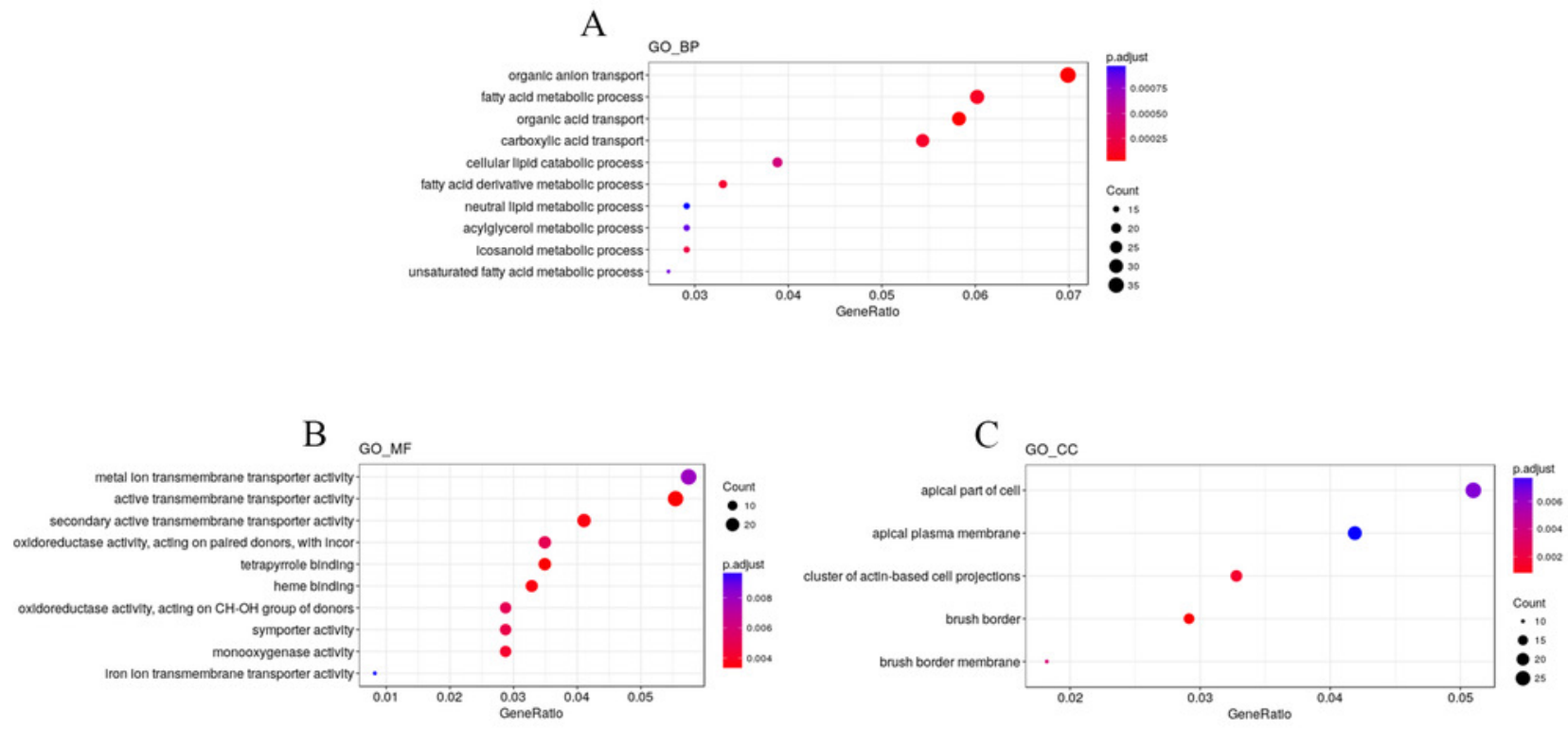
Figure 5

Pathway analysis of the DEGs.

The $x$-axis represents the Rich factor, while $y$-axis represents the name of the pathway. The size of the dot means the number of source genes in the pathway, and the color indicates different FDR values.

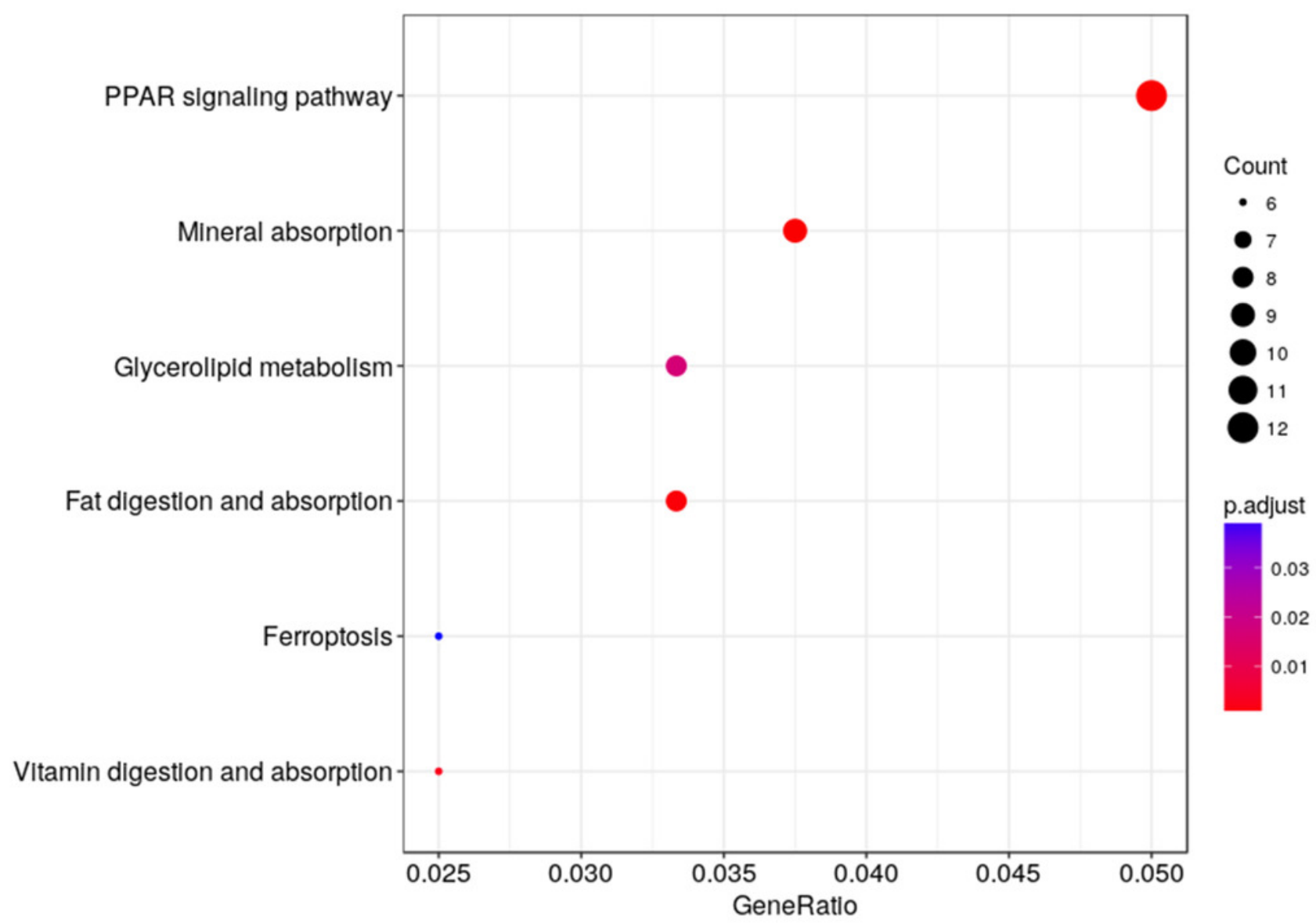


Figure 6

Heatmap of the co-expressed genes.

Three clusters for analysis and k-means clustering method are shown. Most of these genes were down-regulated in 2nd week and up-regulated in 4th week in the first cluster. In the second cluster, most of these genes were down-regulated until the 4th week. In the third cluster, most of these genes were up-regulated from 2 nd week till 4 th week.

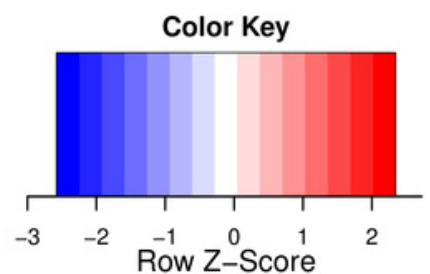

gutS
cogena: kmeans 3

ype of Samples

S W2S W4S

3 Clusters
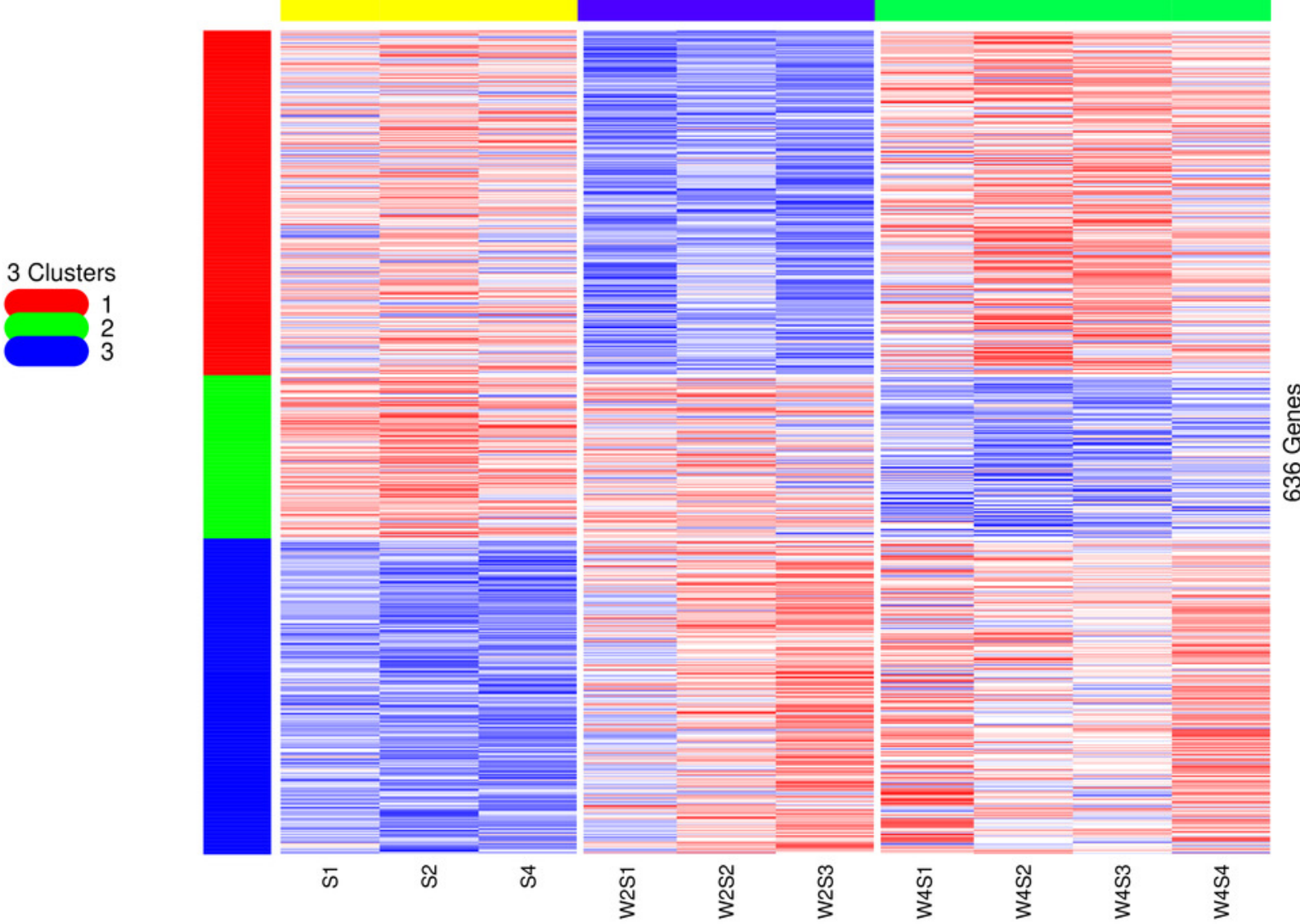
Figure 7

Co-expression analysis of the DEGs.

There are three clusters for analysis and k-means clustering method. Metabolic and digestive related pathways, such as PPAR and renin angiotensin pathway, fatty acid metabolism, are only enriched in the third cluster. 


\section{guts}

\section{cogena: kmeans 3}

kegg_ppar_signaling_pathway-

kegg_renin_angiotensin_system-

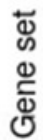

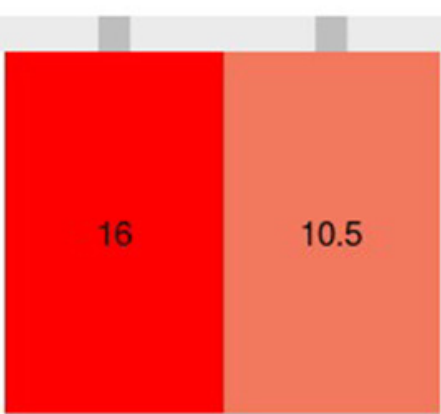

4.7

3.2

score

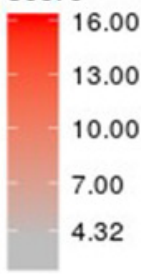

4.5

2.5

2.2

4.5

kegg_axon_guidance-
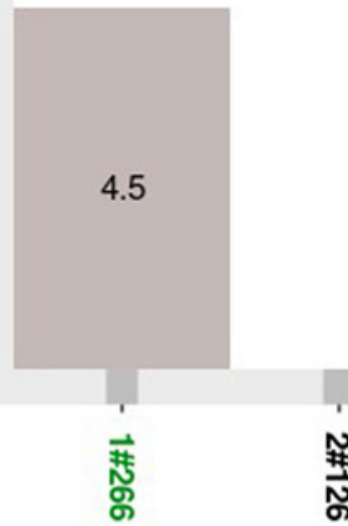

Cluster 


\section{Figure 8}

\section{The most changes genes}

The most changes genes including the top 20 genes with increased expression and top 20

with decreased expression has been listed. A up-regulated genes in different group. B down-

\section{regulated genes in different group.}

A

\begin{tabular}{|cccccc|}
\hline W2S vs. S & logFC & W4S vs. S & logFC & W4S vs. W2S & logFC \\
\hline Hprt1 & 4.670615129 & LOC100909857 & 5.742349393 & F10 & 5.795737241 \\
\hline S100a8 & 4.544846837 & Clec2e & 4.214662004 & LOC103690044 & 4.606522436 \\
\hline Retn & 4.420170943 & Slc19a3 & 4.213854963 & Idol & 4.48267405 \\
\hline Tpsabl & 4.41357889 & Prss35 & 4.205444157 & Ill3ra2 & 4.35541447 \\
\hline LOC100909857 & 4.175303929 & RGD1560608 & 4.196116988 & Hamp & 4.194129504 \\
\hline Slc19a3 & 3.818320081 & LOC103694874 & 3.981314559 & Mybpcl & 4.113211691 \\
\hline RGD1564463 & 3.745113059 & Ace2 & 3.893451696 & Mcpt3 & 4.001366281 \\
\hline Cxcr2 & 3.743506819 & LOC103694864 & 3.763647455 & Mcpt4 & 4.001366281 \\
\hline Asz1 & 3.682479073 & Hamp & 3.705358749 & Mcpt411 & 4.001366281 \\
\hline LOC689230 & 3.609173536 & Idol & 3.7037677 & RGD1560608 & 3.913574647 \\
\hline Trpm8 & 3.557783454 & Cyp2d5 & 3.668249008 & Matn4 & 3.664745814 \\
\hline LOC103691893 & 3.539354977 & Lrrc23 & 3.657985515 & RGD1307603 & 3.613022539 \\
\hline Alpp & 3.45614209 & Ill3ra2 & 3.627318511 & Lpal2 & 3.430919913 \\
\hline LOC100911949 & 3.380532928 & LOC103694855 & 3.59457859 & Asb18 & 3.40239706 \\
\hline Alox15 & 3.359715671 & Plekhs1 & 3.593371138 & LOC100361556 & 3.352460365 \\
\hline Akrlc2 & 3.315860749 & Orm1 & 3.58605829 & LOC100910656 & 3.352460365 \\
\hline Plin1 & 3.2930187 & Tpsab1 & 3.450131116 & Tnp2 & 3.312155111 \\
\hline LOC103694855 & 3.266727129 & Aszl & 3.438258796 & Htr5b & 3.28189709 \\
\hline Rorc & 3.188063043 & Reg3b & 3.397442277 & Pat12 & 3.241844388 \\
\hline Apoal & 3.039792041 & LOCl00361556 & 3.387621905 & LOC685699 & 3.17179547 \\
\hline & & & & & \\
\hline
\end{tabular}

$\mathrm{B}$

\begin{tabular}{|c|c|c|c|c|c|}
\hline W2S vs. S & logFC & W4S vs. S & logFC & W4S vs. W2S & logFC \\
\hline LOC108348322 & -4.757590572 & Ibsp & -6.154120005 & Ibsp & -6.710524845 \\
\hline Asb18 & -3.881572899 & Cavin4 & -3.472180223 & Retn & -6.40588519 \\
\hline Drdl & -3.640204716 & Slpi & -3.412482857 & Plin1 & -5.94920674 \\
\hline Rgs22 & -3.556452287 & Slc27a5 & -3.243128475 & LOC103691893 & -4.23308919 \\
\hline Matn4 & -3.397137083 & LOC688801 & -3.171862892 & Hprl & -3.771013096 \\
\hline F10 & -3.327574306 & Gif & -2.934078283 & Tf2 & -3.549530656 \\
\hline Fam170b & -3.290279134 & Ccr3 & -2.852001359 & Sl00a8 & -3.214851448 \\
\hline Nrsn2 & -3.264771447 & Cldn24 & -2.850291029 & RGD1564463 & -3.189005706 \\
\hline LOC688801 & -3.051405271 & Efcab3 & -2.759019406 & Ms4a4a & -3.129507173 \\
\hline Lpal2 & -3.048100197 & Dnah8 & -2.749643536 & Clec4b2 & -3.115270705 \\
\hline DII3 & -3.037806441 & Plin1 & -2.65618804 & Cebpe & -3.036890294 \\
\hline Gabra3 & -2.954094576 & Clip3 & -2.644709559 & Slc27a5 & -3.015990562 \\
\hline Fam134b & -2.838845767 & DIl3 & -2.63183443 & Rhd & -2.957472611 \\
\hline Atp6v0d2 & -2.820170293 & RGD1563231 & -2.565429351 & Efcab3 & -2.948814766 \\
\hline Cldn24 & -2.803474038 & Avprlb & -2.551919081 & LOC102552988 & -2.94022252 \\
\hline Oas1g & -2.787548925 & Col19al & -2.527188782 & Ccr3 & -2.912765216 \\
\hline Lypd6 & -2.716227135 & Clec4b2 & -2.50241607 & Akrlc2 & -2.903667125 \\
\hline Got111 & -2.709108701 & Pak7 & -2.50093748 & Cavin4 & -2.765103273 \\
\hline Slc9b2 & -2.688120264 & Tp63 & -2.489935723 & Alox15 & -2.712305497 \\
\hline Zfp286a & -2.664513223 & Mtnrla & -2.329810551 & Irx2 & -2.690396992 \\
\hline & & & & & \\
\hline
\end{tabular}




\section{Figure 9}

\section{Gene transcription}

Top 5 differentially expressed genes for each group including the up-regulated gene and down-regulated genes were been detected through RT-PCR. A the up-regulated genes of W2S group compared with S group. B the down-regulated genes of W2S group compared with S group. $C$ the up-regulated genes of W4S group compared with S group. D the down-regulated genes of W4S group compared with S group. E the up-regulated genes of W4S group compared with W2S group. F the down-regulated genes of W4S group compared with W2S group. $* \mathrm{P}<0.05$. 
A

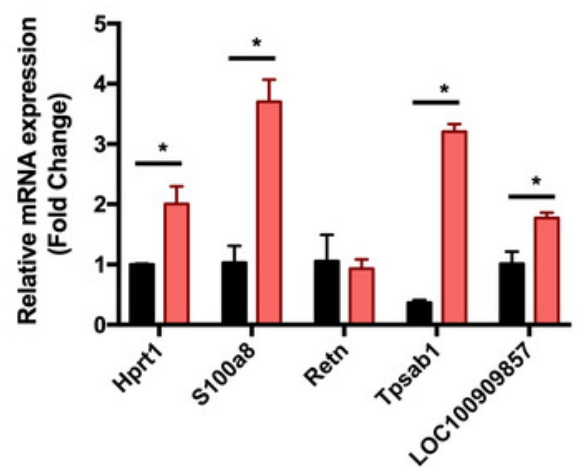

C

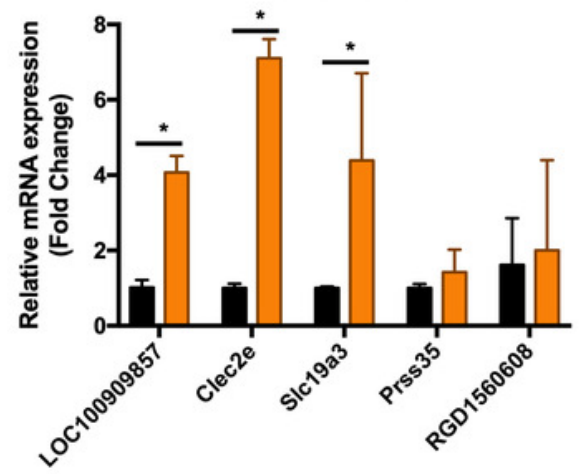

$\mathbf{E}$

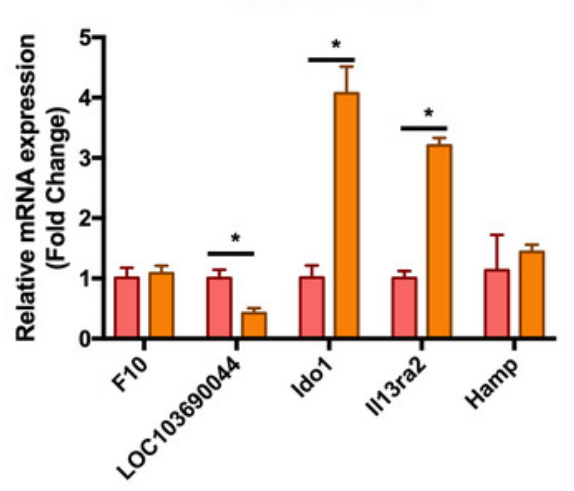

B

W2S vs. S

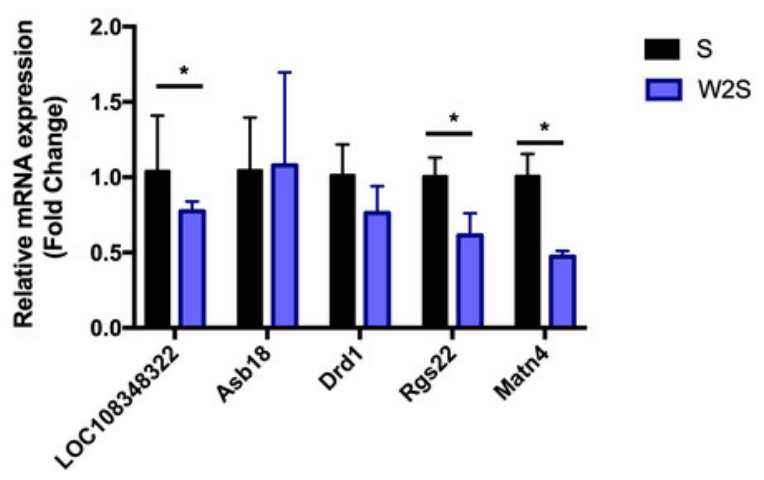

D

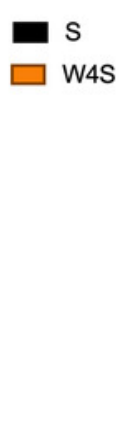

$\square$ W2S
$\square$ W4S

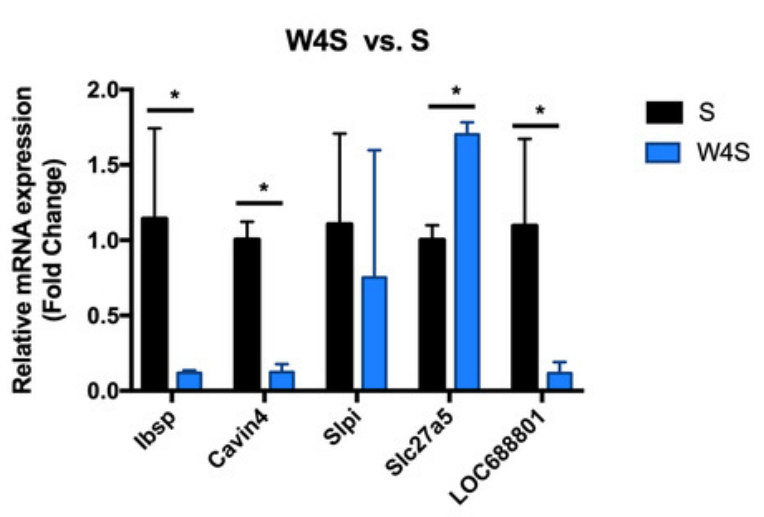

F

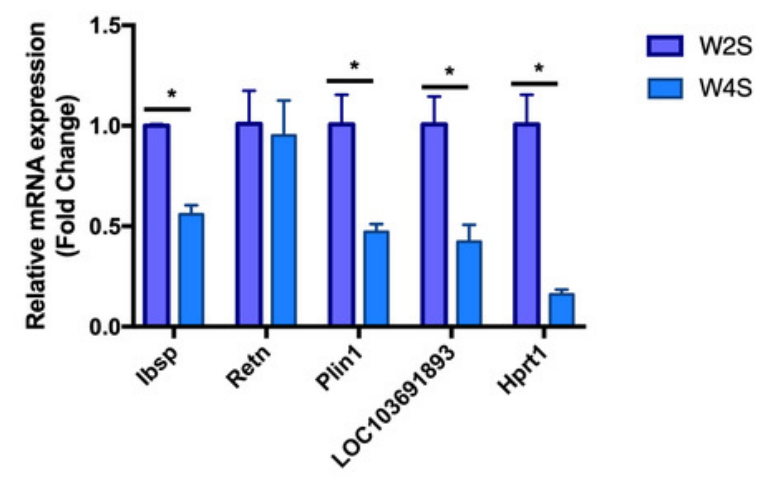




\section{Table $\mathbf{1}$ (on next page)}

Differential gene expression in W2S and W4S

The detailed genes up-regulated and down-regulated in W2S and W4S group. 


\begin{tabular}{|c|c|c|}
\hline Names & total & lements \\
\hline $\begin{array}{l}\text { W2S up W4S } \\
\text { down }\end{array}$ & 4 & Tff2 Plin1 Retn Spaca5 \\
\hline $\begin{array}{l}\text { W2S up W4S } \\
\text { up }\end{array}$ & 120 & $\begin{array}{l}\text { Acadm RGD1304770 Rbm11 Plekhs1 Nyap1 Nat8f4 Apoc2 Ces2h Gpat3 Prss35 Bst1 Lin28a Gdf9 Usp2 } \\
\text { LOC689065 Slc13a2 Epb4113 Slc15a1 Trpm8 LOC100911440 Chdh Adamts15 Bdkrb1 Clec2e Ace2 Cyp2d5 } \\
\text { Akr1c14 Amn Cyp2d4 B3gnt4 Asic3 Slc19a3 Pla2g12b Adam3a Slc43a2 Tmco2 S100a8 Tmem235 Fabp1 } \\
\text { LOC498236 Fmo5 Iyd Alpp Mt1 Cyp2d3 Wfdc21 LOC500948 Dgat1 Apob Trim58 LOC103694874 Tmem37 } \\
\text { LOC100909857 Cxcr2 Phlda2 Fabp2 Exoc314 Gucy2g Tmem120a Cyp4f39 Prr15 Anpep Slc5a11 Akr1b8 Mt2A } \\
\text { Faim LOC691083 Apoa1 Hsd17b11 Slc12a3 Krt15 LOC100912163 Rorc Creb313 Gpt Tsku LOC498424 Bmp8b } \\
\text { Mir194-2 Samd8 Adam32 Erich4 Prss12 Mfsd2a Fut7 Gcnt7 Pdzd3 Akr1b10 Cyp2d1 Reg1a Slc6a19 Tmem86a } \\
\text { Slc7a7 Mme Gsdmd Tssk5 LOC689230 Moxd2 LOC100911949 Btn15 Dusp9 Cyp2j4 Slc25a22 LOC103694864 } \\
\text { Tmem45b Xpnpep2 Asz1 Bdnf LOC100910833 Mall LOC103694855 Tpsab1 Cyp3a9 Areg Pdzk1 Slc23a1 } \\
\text { Acs15 Slc30a10 Ahcy12 Cdhr5 }\end{array}$ \\
\hline $\begin{array}{l}\text { W2S down } \\
\text { W4S down }\end{array}$ & 52 & $\begin{array}{l}\text { Ccdc173 C1q13 Tmem158 Ppp1r42 LOC100911486 Megf6 Drd1 Dnah8 Pak7 St18 Ly6g6e D113 RGD1563354 } \\
\text { Ly6g6d Aard LOC688801 Kcne5 Gba3 Gif Rag1 Nkx1-2 Gabra3 Sp7 UST4r Mzb1 Hmx2 Cacng6 Spata17 } \\
\text { Lrrc3b Col19a1 Cldn24 Vsig4 Mtnr1a Lrrc15 Prss57 Nme5 Bean1 Nrsn2 Cfc1 Fam170b Fabp12 Krt81 Cyp26b1 } \\
\text { Rgs22 LOC108348322 Kcng1 LOC690276 Tnfrsf17 Clca5 Klf8 Dppa3 Alkal1 }\end{array}$ \\
\hline $\begin{array}{l}\text { W2S down } \\
\text { W4S up }\end{array}$ & 19 & $\begin{array}{l}\text { Stra6 LOC685699 Mcpt411 Rln3 Kirrel2 F10 RGD1307603 Uox Clec7a Prrt4 Bbox1 LOC103690044 Mcpt4 } \\
\text { Mybpc1 Oacyl Mcpt3 Lpar4 Htr5b Gjb5 }\end{array}$ \\
\hline W2S up & 71 & $\begin{array}{l}\text { Ly75 Ptgr1 Calcr LOC103691893 B3gnt3 Acox1 Akr1c2 LOC103690017 Adam22 Bche RGD1564463 Ddx43 } \\
\text { Dap11 Mdh2 Tnfaip2 Pabpc11 NEWGENE_620180 Alas2 Trem14 Plin2 Lgals4 Igsf21 Cry1 Upp1 Gsta4 Tldc2 } \\
\text { Hpgd Ms4a4a Hprt1 Cd209f Adamts16 Otop1 Gpd1 Hba1 Nqo1 Aldh3a2 Il1r2 Sbp Idh1 RGD1561777 Mir223 } \\
\text { Slc26a6 Cdkn2b Hmox1 Slc40a1 Hcn3 Abhd6 Sun3 Xpnpep1 Adtrp Gramd2 LOC289035 LOC100910401 Pk1r } \\
\text { Aldob Slc27a4 Irx2 Pbld1 Alox15 Naprt Hba2 Gckr Wfdc3 Knop1 Slc11a2 Sult1b1 Cyp27a1 LOC102548472 } \\
\text { Rhd Gemin711 Reg4 }\end{array}$ \\
\hline W2S down & 152 & $\begin{array}{l}\text { Arhgap44 Eno4 Kcnd3 Slc2a12 Bend5 Syt2 Vegfd Chn1 Sarm1 Dusp27 Elovl2 Abcg4 Pla2g5 Scrn1 Dusp1411 } \\
\text { Mamdc2 Nrep LOC100909776 Enkur Adam23 Ldb2 Ccdc116 Tex52 Klhl32 Nup62c1 Ppp2r2b Plp1 Gpr162 } \\
\text { Slit2 Ndufa412 Lims2 Plekhb1 Grin2a Terc Calhm5 Atp6v0d2 Lrtm2 Snai3 Fmod Cspg5 RGD1359449 Ccdc3 } \\
\text { Sh3tc2 Sema3e Cuzd1 Fam134b Sez6 Rfx2 Mustn1 Matn4 Syt15 Lrrc17 Hspb8 Moxd1 Trpc4 Dusp10 Cdh3 } \\
\text { Adhfe1 Pdia5 Tmprss5 Dleu7 Ptprz1 Mapk4 RGD1311744 RGD1309651 Ctnna3 Igfals Cttnbp2 Prmt8 } \\
\text { RGD1564899 Mreg Dusp1 Lpal2 Armcx6 Slc35f1 Sapcd1 LOC103690028 Chrna4 Unc5a Fxyd6 Syt17 Myh6 } \\
\text { Fam198a Sorbs2 Pkhd111 Thbs2 Got111 Tnfrsf19 RGD1307537 Prelp Tnp2 Spata20 Efhd1 Cdh22 Snhg11 } \\
\text { Atp2b4 B3gat1 Fam198b Otog1 Plxnb3 Stum Zfp286a Nkx6-3 Nr1d1 Lrrc10b RGD1305645 Colca2 } \\
\text { RGD1561102 Klhl38 Pygm Tspan18 Capn6 Tmem200a Hist1h1d Lypd6 Alp1 Lgi4 Ptgfr Rab38 LOC102551716 } \\
\text { Trim6 Cntn2 Pat12 Oas1g Itga7 Osbp2 Ak4 Abi3bp Rp131 Ephb1 Kcnma1 Nlgn3 Arx Mx1 Chrd11 Wnk2 Tppp3 } \\
\text { Panx2 Dact3 Calhm4 LOC103689968 Ntm Tspan11 Ankrd6 Asb18 Adgrb2 Zkscan2 Slc9b2 Myh10 Tspan2 } \\
\text { Arhgef25 Slc29a4 }\end{array}$ \\
\hline W4S down & 02 & Tmco3 Calb1 Sall4 Rgn Esr1 Gpr81 Srl Coch Hsph1 Pcdhb12 Mir6333 Slpi Snai1 Hs3st6 RGD1565767 \\
\hline
\end{tabular}




\begin{tabular}{|l|l|l|}
\hline & & $\begin{array}{l}\text { LOC100910275 F13b Gjc3 Lrrc55 Zfp2 Olr1588 Clip3 Slitrk6 Slc26a7 Ibsp Cebpe Cxc12 Tcf21 Tp63 Tac4 } \\
\text { Pcdh12 Slc27a5 Magee2 Plekhd1 Ccr3 RGD1563231 Clec4b2 Avpr1b Shisa2 Trim72 Rbm46 Pi15 Sim1 Efcab3 } \\
\text { LOC102552988 Wfdc2 Kantr Trpa1 Ptch1 Cyp4f18 Ccr10 Fcer1a Nppc Lancl3 Cavin4 Ednra Ncan I136b } \\
\text { RGD1564801 Sstr2 Alox12 LOC100361018 }\end{array}$ \\
\hline W4S up & 116 & $\begin{array}{l}\text { Wdr63 I113ra2 RGD1565212 Kiss1 Mir143 Ciart Hist1h4m Trpm6 Gpr17 Foxh1 Camk2b Padi3 Bcar3 Asb14 } \\
\text { Car4 RGD1560608 Socs1 LOC103693823 Aplp1 Fam214b Slc52a3 Scn4b LOC100361556 Tubb1 C3 Ugcg } \\
\text { Dnajb3 Mir1b Susd2 Neurod4 Rfk Ky I110 I14r Pex11a Hist2h2ab Mir142 Cnksr1 Lipc Arhgap26 Mir193a } \\
\text { Acot11 Tmem211 RGD1310507 Ccnj Fam71f1 NEWGENE_1306399 Reg3b Abcb4 Abhd3 Olr1 RGD1564606 } \\
\text { Acox1 Lrrc23 LOC102550375 Hist2h2ac Mir23b Gh1 Adgrf3 Noct RGD1309110 Iqcf1 Abcb1a Slc6a8 Abhd4 } \\
\text { Gstm61 Rab24 Lamb3 Mxd3 Mir342 Tnip1 Slc2a9 Pomp Ido1 Tctex1d1 Sbspon Tmem232 Lpin2 Tnnc2 Rnf225 } \\
\text { Mir339 Orm1 Cntn6 Tmem95 Sec16b Lca51 Ezr LOC367195 LOC103689920 Tbx18 Gpcpd1 B4galt6 Abcb1b } \\
\begin{array}{l}\text { Zc3h12a Steap4 LOC103689961 Csrnp1 Dgka Prr13 LOC100910838 Tsacc Unc5d LOC103693384 } \\
\text { LOC100910656 Cmtm2a LOC100364769 Plk3 Grm3 Vpreb2 LOC500684 Matn1 Hamp Paqr7 Fam151a Cldn11 } \\
\text { Lrrn4 }\end{array}\end{array}$ \\
\hline
\end{tabular}

1 